\title{
Ethnologies
}

\section{La captation patrimoniale des savoir-faire horlogers au risque de leur transmission}

Hervé Munz

Volume 36, numéro 1-2, 2014

Patrimoine culturel immatériel

Intangible Cultural Heritage

URI : https://id.erudit.org/iderudit/1037613ar

DOI : https://doi.org/10.7202/1037613ar

Aller au sommaire du numéro

Éditeur(s)

Association Canadienne d'Ethnologie et de Folklore

ISSN

1481-5974 (imprimé)

1708-0401 (numérique)

Découvrir la revue

Citer cet article

Munz, H. (2014). La captation patrimoniale des savoir-faire horlogers au risque de leur transmission. Ethnologies, 36(1-2), 359-382.

https://doi.org/10.7202/1037613ar
Résumé de l'article

À partir d'une ethnographie de la transmission du métier d'horloger réalisée dans diverses écoles professionnelles de l'Arc jurassien suisse, cet article entend critiquer le paradigme de " patrimoine culturel immatériel » et l'ambition de sauvegarde des « savoir-faire traditionnels » qu'exprime la Convention pour la sauvegarde du patrimoine culturel immatériel (2003) de l'UNESCO. La description de caractéristiques propres à la didactique professionnelle et à la manière dont les acteurs qui la mettent en oeuvre qualifient ce qu'est une compétence en horlogerie permet tout d'abord de montrer que la Convention ne peut pas constituer un opérateur de transmission du savoir horloger. En deuxième lieu, il convient de relever que la figure de la transmission que la Convention met en exergue est anthropocentrée et que les dynamiques d'apprentissage du métier dans le cadre scolaire l'invalident. Elles font en effet des objets d'importants opérateurs de transmission de la gestualité et des techniques horlogères. Enfin, l'auteur fait voir que la distinction entre paradigme pédagogique (basé sur un impératif de transparence) et paradigme initiatique (qui fait la part belle aux secrets) ne permet pas de caractériser l'apprentissage dans le cadre des écoles d'horlogerie. La transmission horlogère s'opère également dans des pratiques de voilement/dévoilement sans que l'explicitation des savoirs en soit toujours une condition, ce qui contrarie fortement le principe de sauvegarde qu'incarne la Convention. 


\title{
LA CAPTATION PATRIMONIALE DES SAVOIR-FAIRE HORLOGERS AU RISQUE DE LEUR TRANSMISSION
}

\author{
Hervé Munz \\ Université de Neuchâtel
}

« On ne sauvegarde pas l'horlogerie, on l'exerce. »

Damien, horloger

\section{Introduction : le souci des savoir-faire}

Si les savoir-faire techniques et artisanaux ont une très longue histoire (Pellegrin, 1991), le souci de sauvegarde et de pérennité qui les investit, les (en)cadre et les rend aujourd'hui problématiques dans les espaces publics et privés de nombreux pays du monde est néanmoins récent. De manière significative, en Europe de l'Ouest, les organismes, initiatives et projets dévolus à « l'identification », la « mise en mémoire », la « conservation », la « protection » de ces savoir-faire n'ont massivement émergé que depuis une quarantaine d'années. Ce souci n'est bien évidemment pas né à ce moment-là. Les "métiers » et l'artisanat avaient déjà fait l'objet de diverses stratégies de défense, en réaction à l'essor de la production industrielle jugée morecellante et appauvrissante, durant la seconde moitié du XIX ${ }^{e}$ siècle. En témoignent, par exemple, l'ouverture d'un certain nombre d'écoles professionnelles en Suisse, la création de l'Arts and Crafts Exhibition Society à Londres (1888) ou encore, en France, de la Société d'Encouragement aux Arts et à l'Industrie (1889) qui, changeant de statut et de nom, deviendra la Société d'Encouragement aux Métiers d'Art en 1976 puis l'Institut National des Métiers d'Art en 2010.

Si ce souci des savoir-faire n'est pas apparu en tant que tel, au cours des années 1960, à compter de ce moment-là toutefois, l'ampleur qu'il revêt, la place qu'il accorde à la question de la « sauvegarde » et le décrochement qu'il exacerbe entre l'artisanat et l'industrie le singularisent considérablement. En effet, la mécanisation et la parcellisation accrues du travail industriel, 
la mise en place des premières chaînes de montage automatisées, la standardisation des produits qui apparurent durant les « trente glorieuses » préoccupèrent alors de nombreux acteurs sociaux. Le double constat de la déqualification et de la disparition de certains métiers actualisa, pour le domaine de l'artisanat, le " paradigme des derniers » (Fabre, 2008) et précipita le déploiement d'une « rhétorique de la perte » des techniques anciennes. La perception des menaces imminentes qui pesaient sur ces pratiques fit alors retentir les "sirènes de l'urgence » (Gonseth et al., 2011 : 132-153) et conduisit à la démultiplication massive des initiatives de valorisation et de transmission des «petits métiers » ou des savoir-faire considérés comme "traditionnels ». Depuis lors, ces initiatives ont été progressivement soutenues par une myriade d'acteurs relevant des milieux associatifs, scientifiques, éducatifs, professionnels et en l'espace de quelques décennies, elles ont été prolongées par l'action d'institutions internationales telles que l'UNESCO qui contribuèrent à renforcer la place qu'occupent désormais les savoir-faire dans la matrice patrimoniale qui circule sur la scène mondiale. Pour s'en convaincre, il n'est que de considérer la Convention pour la sauvegarde du patrimoine culturel immatériel (ci-après la Convention) que la Conférence générale de l'UNESCO a adoptée en 2003, concrétisant ainsi trois décennies de pourparlers diplomatiques en matière de politique culturelle mondiale. Cette Convention définit, en effet, le «patrimoine culturel immatériel » (ci-après $\mathrm{PCI})$ comme :

les pratiques, représentations, expressions, connaissances et savoir-faire (je souligne) - ainsi que les instruments, objets, artefacts et espaces culturels qui leur sont associés - que les communautés, les groupes et, le cas échéant, les individus reconnaissent comme faisant partie de leur patrimoine culturel (UNESCO, 2003: 2).

Elle présente, en outre, les «savoir-faire de l'artisanat traditionnel » comme l'un des cinq «domaines » dans lesquelles se manifeste le PCI et en fait une priorité de son programme officiel de protection. Par ailleurs, l'adoption de cette Convention en 2003 vint parfaire le programme des Trésors humains vivants que l'organisation onusienne avait lancé en 1994. En partie conçu comme une traduction du système des Trésors nationaux vivants que le Japon a mis en œuvre depuis 1950, ce programme était destiné à encourager les États membres de l'UNESCO « à accorder une reconnaissance officielle à des détenteurs de la tradition et des praticiens talentueux, ainsi qu'à assurer la transmission de leurs connaissances et savoir-faire aux jeunes générations ${ }^{1}{ }^{»}$.

1. Site Internet de l'UNESCO à l'adresse : http://www.unesco.org/culture/ich/index. php?pg=00061\&lg=FR (consulté le 22 mai 2012). 
S'il est évident que l'UNESCO a joué et joue encore un rôle particulièrement décisif dans l'apparition et le maintien de ce «souci des savoir-faire » et dans la diffusion d'un modèle de prise en charge de «l'artisanat traditionnel », la qualification des pratiques de savoir que l'appareil onusien induit posent un certain nombre de problèmes à l'anthropologie des compétences (skills) et des techniques. Dans le cadre de ma thèse de doctorat, je décris le cas particulier de la Suisse qui a ratifié la Convention en juillet 2008 et m'intéresse à une activité technique qui s'exerce sur le territoire helvétique et se voit régulièrement (re)présentée par les pouvoirs publics, les offices de tourisme et les firmes comme une « tradition » emblématique et un témoin de la « culture technique », à savoir l'horlogerie. Mon travail ne consiste pas à déterminer si la pratique horlogère relève ou non d'une forme de patrimoine immatériel. A contrario, il m'engage à mettre la Convention en perspective en la confrontant aux modalités effectives d'exercice et de transmission du métier d'horloger. Dans le présent article, la mobilisation d'un certain nombre de matériaux collectés durant les phases d'observation et d'entretien que j'ai effectué dans diverses écoles d'horlogerie de l'Arc jurassien suisse ${ }^{2}$ me permettra d'envisager les problèmes majeurs que pose la patrimonialisation (la «PCIsation » devrait-on dire) de la pratique et de la formation horlogère. Bien qu'elle soit théoriquement engagée dans la sauvegarde des savoir-faire, la Convention constitue-t-elle un opérateur de transmission et de pérennisation de ceuxci ? À travers la caractérisation succincte de certaines manières dont la technique horlogère se transmet dans le cadre scolaire, j'effectuerai ici, selon trois axes de problématisation distincts, une critique détaillée des figures de «savoir-faire » et de «transmission » qui se dégagent de la Convention, ainsi qu'une réflexion sur les injonctions à l'explicitation et à la mise en visibilité des savoirs que celle-ci implique.

\section{Le paradigme de " patrimoine culturel immatériel " en question}

\section{La pratique horlogère et le paradigme de $\mathrm{PCl}$}

L'examen succinct des formes actuelles de pratique horlogère dans l'Arc jurassien suisse fait apparaître quatre premières zones problématiques avec la Convention. En effet, la catégorie de « domaine » à travers laquelle cette dernière classe et prend en charge l'univers de la technique n'est pas un cadre de caractérisation qui sied à l'activité horlogère. Dans des travaux antérieurs (Munz, 2011), j'ai eu l'opportunité de montrer en quoi cela

2. Région qui s'étend de Genève à Schaffhouse. 
faisait problème. Premièrement, la «tradition », dans l'horlogerie suisse, est une invention récente. Son apparition est ce qui permit, entre autres, la « renaissance ${ }^{3}$ » de l'industrie helvétique de la montre mécanique et son repositionnement dans la gamme des produits de luxe, au début des années 1980. La convocation de cette notion eut alors pour fonction de qualifier et d'authentifier l'horlogerie en l'ancestralisant et en l'ancrant dans le territoire suisse. Deuxièmement, la « tradition » n'est pas artisanale dans la mesure où elle est une pure invention des marques et des groupes industriels qui revendiquent le fait d'en être dépositaires « par héritage » à des fins de valorisation commerciale. Troisièmement, les formes actuelles d'artisanat horloger ne sont pas traditionnelles parce que les acteurs qui s'en réclament ne revendiquent pas nécessairement leur appartenance à la " tradition ", ne se reconnaissent pas dans cette notion ou entretiennent avec elle un rapport ambivalent. Par ailleurs, en définissant le PCI comme ce qui est «transmis de génération en génération », la transmission est implicitement présentée dans la Convention comme une figure continuiste et linéaire, ce que conteste l'histoire récente de l'horlogerie suisse (Munz et Geslin, 2010). Si, aujourd'hui, la réalisation de certaines «finitions main » faites « à l'ancienne » ou de certains mécanismes à " grandes complications » datant des XVIII et ${ }^{\text {XIX }}{ }^{\mathrm{e}}$ siècles est courante, elle n'a été rendue possible, lors du repositionnement de l'horlogerie mécanique sur un segment « haut-de-gamme », qu'à la faveur de la revitalisation de certains « artisanats d'art » alors quasiment oubliés (anglage, guillochage, émaillage, peinture sur miniature, gravure main) ou de la recréation d' « arts de faire »

3. Le terme de « renaissance » désigne ici le retour inattendu de l'industrie horlogère suisse sur la scène internationale après qu'elle a été ébranlée par une importante crise structurelle, à la suite du « premier choc pétrolier » de 1973. À ce moment-là, différents facteurs tels que la hausse significative du franc suisse, l'essor de la concurrence internationale, la perte progressive d'importants marchés tels que les États-Unis et la commercialisation de la montre à quartz par l'industrie nippone fragilisèrent les structures de l'horlogerie suisse, alors centrée sur la fabrication de montres mécaniques (moins précises et plus chères que les montres à quartz) et essentiellement composée de petites structures de production peu compétitives. Ses effectifs passèrent alors, en un peu moins de dix ans, de quatre-vingt-dix mille à trente mille personnes. Dans la première moitié des années 1980, toutefois, la restructuration de la branche, la réapparition d'un intérêt pour la montre mécanique, le repositionnement de cette industrie dans la sphère des produits de «prestige » et le développement de la montre à quartz suisse Swatch conduisirent à la " résurrection » de l'horlogerie helvétique. 
sur des produits de « haute technicité » qui n'étaient presque plus fabriqués (tourbillon, quantième perpétuel, équation du temps, grande sonnerie). Dix ans auparavant, la « crise du quartz » menaçait alors la possibilité même de fabriquer des montres mécaniques et, de ce fait, compromettait grandement la transmission de nombreux aspects du métier. Quant à la période fordiste qui précéda la crise, l'industrie horlogère suisse proposait majoritairement des produits standardisés. Leur fabrication requérait alors une importante mécanisation des procédés qui contribua à raréfier la pratique de techniques anciennes et à fragiliser leur partage. Au début des années 1980, les marques désireuses de « revenir aux origines » et de «perpétuer la tradition des complications » durent alors solliciter des horlogers qui recomposèrent, à tâtons, les savoirs qui sous-tendaient ces produits, en consultant des ouvrages, des photographies, en entreprenant des recherches dans les fonds d'archives et les musées, en s'inspirant d'autres pièces ou en démontant tout simplement d'anciens mouvements à disposition. Dans ces cas-ci, c'est bien une série de discontinuités qui conditionna la relance de ces pratiques et permet désormais la transmission de certaines méthodes de construction mécanique relative à l'« horlogerie précieuse ». L'« artisanat traditionnel transmis de génération en génération » que l'UNESCO entend valoriser à travers la Convention ne correspond donc pas, d'un point de vue théorique, à la pratique actuelle de l'horlogerie dans l'Arc jurassien suisse.

Une ethnographie de la formation horlogère comme critique de I'acception PClste de la "transmission"

Deux constats préliminaires m'ont conduit à focaliser ma recherche sur la notion de «transmission ». D'une part, la Convention lui réserve une place importante puisqu'elle constitue à la fois un critère d'identification du PCI (elle est ce qui permet d'identifier la dimension patrimoniale d'une pratique) et un objectif à atteindre (elle est carcatérisée comme un moyen de sauvegarder le PCI). D'autre part, dans le monde horloger, il y bien évidemment de la «transmission » en jeu en dehors des processus de patrimonialisation. Elle est une catégorie dont la mobilisation fait sens pour de nombreux acteurs qui conçoivent ainsi des moyens qui la favorisent, des obstacles qui la compromettent ainsi que des lieux qui lui sont dévolus. Les écoles professionnelles sont de ceux-ci, raison pour laquelle, fort de ces deux constats, j'ai entrepis d'étudier pendant deux ans les dynamiques de transmission institutionnelle du métier d'horloger en me focalisant sur plusieurs écoles de l'Arc jurassien suisse qui proposent un apprentissage ${ }^{4}$ en

4. En Suisse, l'«apprentissage» constitue la formation initiale que doivent suivre les jeunes gens qui désirent s'orienter vers la pratique et apprendre un métier, 
horlogerie. J'y ai alterné les entretiens semi-directifs avec les directeurs, les enseignants, les apprentis horlogers et les temps d'observation participante en «salles des maîtres » et en " ateliers » où j'ai pris part aux cours de pratique que les apprentis suivaient deux à trois jours par semaine. J'ai choisi de travailler avec les écoles d'horlogerie pour trois raisons majeures. Tout d'abord, en Suisse, la formation professionnelle initiale des horlogers passe inévitablement par l'école. Que ceux-ci choisissent de la faire en mode dual ou non, ils sont nécessairement conduits à effectuer les cours de théorie horlogère et à passer les examens finaux à l'école. Deuxièmement, les entretiens préliminaires que j'ai menés avec différent-e-s horlogers/ères travaillant dans les mondes de la «formation » et de la «profession» m'ont permis de déterminer qu'il existait, entre ces deux mondes, d'importantes tensions relatives à la teneur des qualifications délivrées à l'école ainsi qu'à la durée et au coût des enseignements. Enfin, contrairement au milieu de « l'enseignement » horloger, celui de « l'industrie » est difficile d'accès en vertu des principes de « confidentialité » et des «secrets de métier » qui l'organisent, et je ne voulais pas m'engager contractuellement à signer des clauses de réserve restrictives ou contraignantes avec quelque marque que ce soit.

En envisageant la cognition dans une perspective résolument « située » (Lave, 2011), je me suis intéressé à l'apprentissage horloger en focalisant mon attention sur les formes d'incorporation du savoir et les techniques du corps qu'elles impliquent. J'ai ainsi interrogé la place de la gestualité technique dans les dynamiques de transmission des compétences horlogères. Dans les écoles d'horlogerie, la didactique professionnelle thématise et problématise, de manière centrale, cette question de la transmission. Celleci repose, en partie, sur la reconnaissance des compétences que négocient enseignants et apprentis en rapportant les gestes, les connaissances et les opérations techniques à un « répertoire » relatif au métier d'horloger.

\section{Savoir-faire contre " sens mécanique " horloger?}

Le "savoir-faire horloger » n'existe pas

Un des premiers problèmes que la Convention pose en s'engageant

après avoir terminé la scolarité obligatoire. Pour ce qui concerne l'horlogerie, l'« apprentissage » standard dure trois ans et se déroule en école à temps plein ou en mode dual (les apprentis suivent les ateliers de pratique en entreprises et les cours de théorie à l'école). Il conduit à l'obtention d'un certificat fédéral de capacité (CFC) et au titre d'« horloger praticien ». 
formellement dans la sauvegarde de savoir-faire considérés comme «patrimoniaux » est celui de leur identification. Dans une activité technique, tout n'est jamais patrimonialisable et l'acte de mise en patrimoine suppose nécessairement une restriction et donc un choix préalable. Aussi, en vertu de quels critères fixer, par exemple, des «partages » et des «frontières » au sein du métier d'horloger pour y caractériser le «savoir efficace » (Chevallier, 1991), ce qui en témoigne et ce qui n'en relève pas? En quoi le choix qui préside à l'objectivation du savoir-faire en patrimoine reconduit-il les normes arbitraires, inhérentes au métier? Comment ces normes sont-elles négociées entre une multiplicité de praticien-ne-s? Constituent-elles le résultat de consensus patiemment établis ou le produit des rapports de force aléatoires qui organisent la pratique? Il s'agit là d'insolubles questions pour la cause patrimoniale. Ce d'autant plus que comme n'importe quel « objet de la transmission », le savoir-faire horloger est « introuvable » (Chevallier et Chiva, 1991) tant il se disperse dans une multitude de gestes, de perceptions, de maniement d'objets, de valeurs et de temporalités diffuses (Adell, 2010). En outre, l'industrie horlogère suisse n'est de loin pas composée que d'horlogers et comme le rappelle la Convention patronale de l'industrie horlogère, « la panoplie de métiers de la branche horlogère [étant] très vaste [...], [1]es horlogers diplômés ne constituent qu'un petit pourcentage de l'ensemble des travailleurs ${ }^{5}$ ». Le savoir-faire lié à la fabrication des montres mécaniques et quartz ne se résume donc pas aux connaissances et aux compétences que requiert le métier d'horloger mais se trouve distribué dans d'autres corps de métiers tels que dessinateur, micromécanicien, étampeur, décolleteur, faiseur d'aiguilles, cadranier, boîtier.

Par ailleurs, pour ce qui concerne le milieu de la formation horlogère, mes deux années de «terrain » attestent du fait que, dans les écoles professionnelles, le terme de «savoir-faire » est peu mobilisé par les horlogers pour décrire ce qu'ils font ou ce qu'ils visent. Selon un certain nombre d'entre eux, il n'existe pas de « savoir-faire horloger » au sens général du terme mais des manières de faire, de réfléchir, de construire l'information technique à partir de situations de pratique spécifiques. Penser ou thématiser les registres de la cognition et de l'action ne se fait qu'en regard de confrontations concrètes avec un type de pièce d'horlogerie, un objectif précis, un mécanisme particulier, une fonction à vérifier, un objet à employer, une technique à mettre en ouvre, un problème à résoudre. Les discours qui portent sur les manières d'apprendre et de comprendre

5. Site internet de la Convention patronale de l'industrie horlogère suisse : http://www.cpih.ch/fr/m4/for_et_met/page_4_4_0_4.php [consulté le 4 avril 2013]. 
sont toujours ancrés dans des opérations concrètes. En revanche, dans le monde horloger, le terme «savoir-faire » fait, à plus d'un titre, l'objet de nombreux usages communicationnels, promotionnels et marketing que ce soit dans le discours des marques, des associations horlogères ou des acteurs du développement territorial (villes, musées, tourisme). De tels usages discursifs du « savoir-faire » ont une acception très générale et ne renvoient généralement à rien de précis au niveau de la technique horlogère. Le terme est employé comme synonyme d'expérience, d'habitude et atteste implicitement de la qualité d'un produit, d'un service ou d'un lieu lié à l'horlogerie. La frilosité des horlogers à utiliser ce terme témoigne avant tout du fait qu'il n'est pas un terme de praticien mais un outil de caractérisation de l'horlogerie qui permet à une autre catégorie d'acteurs d'accomplir son travail, à savoir les agents de valorisation de l'industrie horlogère. Considérant l'orientation de la Convention qui fait de la « reconnaissance » un critère de définition du PCI, un tel positionnement des horlogers ne fait-il pas problème ? En effet, pour être identifié comme du PCI, un savoir-faire doit ainsi être reconnu, par les « individus » qui en sont dépositaires, comme leur patrimoine culturel. Ce principe de conscientisation normative ne semble pas convenir à certains praticiens qui ne se reconnaissent pas nécessairement dans ce terme de « savoir-faire ». En contrepoint, les enquêtes conduites avec les membres des secteurs horlogers de diverses écoles professionnelles m'ont permis de relever que le terme qui qualifie régulièrement ce que les enseignants cherchent à transmettre et qui façonne leurs didactiques est celui de « sens mécanique ».

Le "sens mécanique ", une "éducation à l'attention "

Dans le cadre de l'apprentissage horloger, la construction des connaissances et des habiletés techniques s'opère dans le déploiement de diverses formes de didactique professionnelle. Je m'intéresse particulièrement à la manière dont les acteurs qui les organisent, et y prennent part, caractérisent explicitement et implicitement le travail de qualité et définissent ce qu'est une compétence. À partir des observations que j'ai menées dans les classes d'apprentissage et des entretiens que j'ai réalisés avec maîtres et apprentis, je me propose de décrire ici quelquesunes des caractéristiques de ce «sens mécanique ». Tout en refusant de le constituer en totalité et de le réifier en une substance définie une fois pour toutes, j'entends le confronter à l'acception formelle du « savoir-faire » qu'exprime la Convention.

Il n'est, tout d'abord, pas étonnant que les divers acteurs du monde 
de l'apprentissage horloger parlent de «sens » plutôt que de «savoir » pour qualifier les aptitudes qu'ils valorisent. Comme le dit la sociologue Laurence Marti, il y a, en horlogerie « la présence d'une très forte identité de métier [...] [b]asée sur l'importance du rapport direct à la matière, sur l'importance des sens [...]» (Marti, $2001: 10)$. L'injonction « il faut sentir la matière » est de celles que j'ai eu l'occasion d'entendre dans la bouche de nombreux horlogers et horlogères, que ceux et celles-ci travaillent dans le cadre scolaire ou dans le milieu professionnel. Apprendre la technique horlogère c'est, en effet, s'approprier un ensemble de " geste[s] efficace[s] sur la matière " (Leroi-Gourhan, 1981) en les faisant et les refaisant inlassablement. Ces gestes conditionnent la création de « référentiel[s] » (Geslin et Salembier, 2002: 244) qui permettent de déterminer comment procéder et comment atteindre tel objectif technique. Sous l'effet de ces gestes répétés, les apprentis voient leur dextérité se développer en même temps que leurs postures et leurs habitudes sensorielles se transforment. Ces jeunes gens incorporent ainsi progressivement des techniques qui sont des manières de connaître la matière en agissant sur elle et de se constituer en tant que sujets horlogers. Comme le relevait un enseignant, " en observant les étapes par lesquels les apprentis, fille et garçon, deviennent des horlogers, on voit avant tout des corps se transformer ». L'appropriation de ce «sens mécanique » s'inscrit également dans un régime de valeurs relatif à l'activité horlogère. L'inculcation de la gestualité par laquelle le «sens » du métier s'incarne relève de l'apprentissage d'une économie morale de la profession qui fait de l'autonomie et de la responsabilité individuelle des valeurs cardinales, allant de pair. De plus, l'horloger qui a un «bon sens mécanique », doit avoir l'œil « aiguisé », le regard exercé, il doit "savoir voir » (Cornu, 1991). Le « sens mécanique » n'est pas présenté comme un sens intuitif même si certains praticiens parlent de «feeling » « il faut sentir les choses! »), il est avant tout un sens « réflexif » fondé sur une capacité à observer. Le développement du sens de la vue, l'amélioration de la qualité de l'observation, l'incorporation de techniques du regard spécifiques « sans quoi il ne peut y avoir de bonne réflexion en amont du geste technique », sont des lignes directrices qui accompagnent les apprentis au quotidien. «Apprendre à regarder », à « voir » signifient choisir, croiser et confronter les informations pertinentes pour tramer les prises de décision et agir en pouvant justifier ses actions. Ces techniques permettent de construire ce que Grasseni (2004) appelle une «vue outillée » (Skilled Vision), un «sens » du regard qui opère comme un « savoir visuo-moteur » (Visuomotor Knowing, Downey, 2007) et sert de repères dans les « services complets » qui sont effectués sur les différents mouvements horlogers. Ce « savoir visuo- 
moteur » est entraîné par des exercices répétés de familiarisation avec des techniques de représentation des formes (dessin technique, conception de croquis, de schémas). Il doit conduire à « toucher les choses avec les yeux » et à développer la capacité de les « visualiser ». La visualisation est ce qui conditionne la réussite des processus de résolution de problèmes en permettant au praticien de se projeter dans l'espace et d'anticiper les effets qu'aura tel de ses gestes, telle de ses opérations. Ce qui caractérise le mieux le «sens mécanique » horloger est sans doute cette aptitude à résoudre un large spectre de problèmes spécifiques plus que la détention de connaissances particulières (même si cette aptitude en implique, concernant les matériaux, par exemple) ou de savoirs procéduraux aux contours nets. Le « sens mécanique » correspond ainsi moins à l'expression d'une forme de maîtrise technique répétable à souhait qu'au fruit d'un apprentissage horloger qui se conçoit et se vit comme une graduelle « éducation à l'attention » (Ingold, 2000). L'attention seule permet la compréhension des systèmes techniques ainsi que la formation d'un sens de l'adaptation, une compétence en matière d'ajustement du savoir au besoin de la situation, quitte à faire preuve d' " imagination ».

Il est cependant intéressant de souligner que les enseignants horlogers caractérisent le "sens mécanique » en utilisant un champ lexical qui relève explicitement de l'intellect et de la rationalité. Ils emploient, en effet, des termes tels que «logique », " analyse », " réflexion », «méthode » et « rigueur » pour le qualifier, ce qui prouve la forte tendance à l'intellectualisation de la pratique horlogère. Un enseignant horloger concédait que ce «sens » est « ce qui relève de l'analyse et ne peut être automatisé! » et rejoignait les propos que me tint un horloger établi à son compte depuis nombreuses années : « on pense que l'horlogerie est un métier manuel, c'est faux. Moi j'ai toujours revendiqué qu'il s'agissait avant tout d'une activité intellectuelle! »Si le « sens mécanique » se traduit bien dans un savoir du corps qui se fonde effectivement sur une synesthésie, une complémentarité des facultés " de toucher ", «d'observer ", « d'écouter » - en somme sur des manières de rendre le système perceptif disponible - il suppose une importante part de "réflexion » et, n'en déplaise à certaines théories de l'incorporation, de « réflexivité » (il est souvent demandé aux apprentis de savoir exactement ce qu'ils sont en train de faire). L' « habileté technique », la "dextérité » ne suffisent ainsi pas à définir le «sens mécanique ». Leur acquisition est une condition nécessaire mais non suffisante à l'appropriation de celui-ci. À ce titre, certains enseignants considèrent que les praticiens qui sont seulement extrêmement habiles de 
leurs mains peuvent faire de «bons opérateurs » mais ne font pas de bons horlogers car ils ne sont pas capables de "réfléchir » certains problèmes techniques et d'en « imaginer » des solutions. L'aisance manuelle n'est ainsi qu'une partie de ce qui est assigné sous le terme de «sens mécanique ». En revanche, il est toujours corrélé à une forme d'expérience directe avec la matière. Il ne peut s'acquérir autrement qu'à travers l'exercice et la pratique continue de l'horlogerie, et ne peut équivaloir aux savoirs que déploieraient des ingénieurs qui n'auraient pas suivi une formation préalable par l'établi. Ceux-ci manqueraient immanquablement de « sens du concret », ne saurait pas « gratter », éprouver la matière. Ils n'auraient pas « l'habileté manuelle » qui constitue, dans cette situation-ci, une sorte de signature, une part constitutive du répertoire, un élément central dans l'identification des praticiens et des praticiennes au métier. Pour ceux et celles-ci, cette qualification du «sens mécanique » constitue également une sorte de garde-fou contre la «théorie pure » et les dérives intellectualisantes qui se matérialisent dans de nouveaux produits mal conçus ou dans des solutions inapplicables dont plus d'un horloger se plaint au quotidien. Nombreux sont, en effet, les gens de métier qui s'offusquent de voir désormais les compétences de construction et de design horlogers se concentrer dans des bureaux techniques que composent majoritairement des « masseurs de claviers » qui n'ont plus le « sens » de la faisabilité technique, « ne savent pas tenir un tournevis ou limer plat » et ont perdu tout « contact avec la matière .

L'appropriation du « sens mécanique » ne relève ainsi pas uniquement d'un ensemble de connaissances théoriques et ne se limite pas à la mise en œuvre d'habiletés manuelles. C'est pourquoi, la nature des compétences qui se négocient et se transmettent dans le cadre de l'apprentissage horloger ne correspond aucunement au partage théorie/pratique bien que la formation soit institutionnellement organisée en fonction de celui-ci (il y a effectivement des cours hebdomadaires de "théorie horlogère " auxquels répondent des cours de «pratique » en atelier). Cependant, le savoir qui se met en œuvre dans le cadre scolaire ne peut être rapporté exclusivement ni à l'un, ni à l'autre. Cela fait dire à plus d'un enseignant que « les apprentis qui sont bons en pratique sont aussi ceux qui sont bons en théorie, et inversement ! » Cette hybridité de nature fait du « sens mécanique » « la chose la plus difficile à acquérir ». Il ne s'apprend pas en tant que tel, il s'exerce, s'acquiert progressivement, s'affine, s'affûte. Il est à la fois un prérequis pour suivre la formation d'horloger (les « examens d'entrée » et les « périodes d'essai de trois mois » à l'école ont pour fonction 
explicite de révéler les apprentis qui en sont dotés) et un objectif auquel cette dernière doit conduire.

Ainsi, le processus de mise en place de la Convention conduit toutes les pratiques identifiées comme du PCI à être fichées, décrites, documentées puis objectivées en dossiers afin d'être inscrites dans un inventaire. Le PCI est donc avant tout une « liste» (Hafstein, 2006). Un tel constat autorise à envisager avec suspicion la portée réductrice d'une telle traduction patrimoniale $\mathrm{du}$ «sens mécanique » horloger. Comme nous l'avons précédemment vu, il n'existe pas un objet qui s'appellerait le « savoir-faire horloger » dont la netteté des propriétés et les frontières identifiées une fois pour toutes permettraient de l'isoler, de le décrire sans n'en rien omettre, puis de le traduire en une suite d'énoncés précis qui constitueraient les « instructions de procédure » nécessaires à sa répétition. En ce sens, le « savoir-faire horloger » est introuvable et le «sens mécanique » qui est négocié, partagé et transmis dans les écoles d'horlogerie, résolument diffus et difficilement décomposable en une liste de paramètres fermée. D'autre part, est-il utile de rappeler qu'en codifiant une pratique de la sorte, on ne transfère ni les régimes de valeur qui la caractérisent, ni les cadres et les conditions dans lesquels elle s'exerce? Le « sens mécanique » ne peut être ainsi envisagé qu'en regard des situations où il est effectivement mis en œuvre et, de fait, incorporé. Considérant que « les savoir-faire ne peuvent exister sans les habiletés » (Geslin et Salembier, 2002 : 24), il n’y a pas d'autres espaces de manifestation des compétences que les corps. Aussi, patrimonialiser le "sens horloger » conduirait avant toute autre chose à patrimonialiser des corps en activité. Comment rendre alors compte de ces corps qui se transforment et se trouvent dans un incessant devenirhabile qui a ses propres fluctuations? Le temps fait ainsi partie de cette économie des savoir horlogers et de l'écologie que leur mise en œuvre exige. La «production » d'horlogers est, en effet, un long processus, jamais achevé, d'habituation des corps au métier. Acquérir le «sens mécanique ", s'entraîner à la résolution de problèmes lors de la réparation de pièces s'inscrivent nécessairement dans une durée de l'activité que la patrimonialisation ne peut capter et restituer.

Au demeurant, la transmission du métier n'est pas une répétition de celui-ci mais conduit à son appropriation, à sa réinscription dans de nouvelles coordonnées de pratique. Dans l'apprentissage d'une technique telle que l'horlogerie, il n'y a pas de reproduction mécanique des « tours de main ", d'imitation à l'identique des façons de faire. Même le principe de mimétisme qui façonne un grand nombre de situations d'apprentissage 
suppose toujours une transposition de la gestualité et une réorchestration adaptative de celle-ci. Les corps des apprentis, loin de ne constituer que des « réceptacles » où se répèterait l'écho des gestes à accomplir, sur lesquels s'imprimerait la mémoire de compétences bien circonscrites, acquises une fois pour toutes et ré-activables au besoin, correspondent davantage à des espaces où s'expérimentent les rapports aux objets et aux environnements matériels, où le savoir se traque, s'élabore pas à pas, en piétinant beaucoup, en reprenant, en apprenant à former des repères qui ne sont jamais les bons du premier coup, et à les ajuster, encore et encore. Un métier ne se perpétue donc qu'à la faveur du déploiement d'une série de différences et il n'est pas sûr que la Convention sache faire d'un tel mouvement autre chose qu'un paradoxe qui menace son idéologie de l'identité continuée.

\section{La transmission où la Convention ne peut la penser}

\section{Les objets comme opérateurs de transmission des compétences horlogères}

S'il est vrai que la Convention prend soin d'inclure dans sa définition du PCI « les instruments, objets, artefacts et espaces culturels qui 1[ui] sont associés »(UNESCO, 2003), elle témoigne cependant d'un anthropocentrisme implicite dans sa manière de qualifier la transmission du patrimoine. En précisant que, « transmis de génération en génération, [le PCI] est recréé en permanence par les communautés et groupes [...] », elle décline une figure de la transmission qui s'effectue d'humain à humain et dans laquelle les objets n'ont pas droit de cité, raison pour laquelle il convient ici de faire valoir leur «parti pris ». En effet, dans la pratique horlogère et l'apprentissage du métier, la place qu'occupent les objets est centrale. Les processus cognitifs que la mise en œuvre du « sens mécanique » implique s'élaborent en se distribuant sur un ensemble varié de corps, d'espaces et d'objets (par exemple outils d'établi divers, huilier, réglette, potence, mouvements d'horlogerie, composants, gants à doigt, crayon, feuilles de notes, plans, feuilles de consignes, manuels de théorie, machines de contrôle, etc.). Les corps des praticiens sont ainsi « faits » d'un nombre important d'artefacts qui prolongent, augmentent ou ré-agencent leurs capacités. Il n'est pas possible de caractériser les formes incorporées du savoir horloger sans envisager le rôle de ces objets dans l'activation de la mémoire corporelle des praticiens. Le corps humain (œil, main, dos, coude) est si souvent outillé que certains acteurs du milieu parlent d'« opérations manuelles », par contraste avec les « opérations automatiques », en 
incluant inséparablement, sous cette même formule, la main et un très grand nombre d'outils, voire de machines de travail (l'œil de la loupe oculaire appelée migros, les mains de la brucelle, du tournevis, de la cheville de bois, de la lime, du cabron et même parfois du tour automatique!). La construction de l'identification professionnelle des horlogers ne s'élabore ainsi pas autrement que dans un intime dialogue entre ces différentes formes de matérialités. Dans l'ethnographie de la formation horlogère que je réalise, les descriptions que je dresse de l'activité des enseignants et des apprentis m'engagent à prendre en considération l'ensemble de l'environnement artefactuel qui sous-tend la transmission de la gestualité et l'appropriation des techniques corporelles. Les « choses » qui organisent l'espace des salles de cours et entourent les horlogers sur les établis sont multiples, et apprendre à s'en servir constitue un important enjeu dans la mise en œuvre des compétences. Les maîtres horlogers « traduisent » la technique horlogère en " matière » enseignable, distribuée, tout au long du cursus d'apprentissage, sur de multiples supports matériels (fiches papier diverses, manuels et ouvrages, fiches techniques, plans, notes affichées au rétroprojecteur. dictée de "problèmes » à retranscrire sur papier). De plus, compte tenu de la petitesse des pièces, de l'échelle microscopique à laquelle s'effectue l'activité horlogère et de la précision souvent proche du dixième de millimètre qu'elle requiert pour que les «tolérances des cotes » soient respectées, l'usage d'objets didactiques visant à agrandir le travail en cours est courant (écran relié à un microscope sur l'établi de l'enseignant, réalisation de nombreux croquis, dispositif de projection sur écran pliable à partir d'un rétroprojecteur ou d'un vidéoprojecteur, modèles géants de démonstration). Les explications données par les enseignants à titre d'exemples ne le sont ainsi qu'à la faveur de leur inscription et de leur décomposition dans tout un matériel pédagogique qui vise à faciliter la transmission des habiletés. Comme je le mentionnais déjà dans le premier chapitre, il n'existe, pour les praticiens, pas de « savoir-faire horloger » en général. Les connaissances et les techniques s'enseignent dans des situations de pratique qui les ancrent nécessairement sur des mouvements (ex. mécanique simple pour montres de poche ou montres-bracelets, mécanique automatique, quartz) et des mécanismes d'horlogerie particuliers (ex. remontoir et mise à l'heure, remontoir automatique, quantième, chronographe) ou de composants spécifiques (ex. spirale, balancier, ancre, roues, axes, pignons, pierres, ponts etc.) et des opérations spécifiques (ex. contrôle des ébats de hauteur, partagement des mobiles, équilibrage du balancier). Il s'agit d'une sorte de tête-à-tête que les apprentis mènent avec les pièces d'horlogerie, tête-à-tête encadré par tout un dispositif d'actants qui sont autant de médiateurs dans 
la construction du savoir. Les compétences transmises varient ainsi d'une pièce à l'autre en fonction de leurs particularités, des difficultés qu'elles posent. Il y a là l'exigence d'une adaptation continue et d'une capacité d'ajustement systématique.

Un jour, je confiais à un enseignant les difficultés que je rencontrais à adopter une méthodologie d'observation adéquate pour caractériser les processus cognitifs qui s'élaboraient dans les classes, une méthodologie qui me permette de décrire finement les micro-gestes et les minuscules opérations des horlogers à l'établi sans les déranger et en pouvant toutefois voir ce qu'ils faisaient. Contre toute attente, celui-ci m'avoua se retrouver souvent confronté aux mêmes difficultés que moi : " ton travail touche à quelque chose qui nous fait aussi problème, les difficultés que tu as dans l'observation on les a aussi dans la transmission... comment corriger au mieux afin de transmettre la bonne manière de faire ? Je suis soucieux d'améliorer mon encadrement d'enseignant mais, au bout d'un moment, je me rends bien compte que tout est tellement petit que la démonstration a des limites en termes didactiques! » Le constat de ces « limites » fut moins un aveu d'impuissance qu'une façon de signifier qu'en horlogerie, la transmission ne s'opère pas toujours directement de personne à personne. Les nombreux objets qui façonnent, à la fois, les cadres et les enjeux de l'action technique, et sont parties prenantes de l'élaboration des savoirs, constituent également des médiateurs dans la transmission des compétences horlogères. La figure humaine du maître décomposant et expliquant le geste pour mieux le transmettre n'est donc pas toujours centrale dans l'ajustement du corps aux exigences de précision propres à l'activité et dans la mise en oeuvre d'une méthode de travail adéquate. L'encadrement didactique assumé par la présence et le regard des enseignants est prolongé, médiatisé dans les objets, voire bien souvent délégué à ceux-ci. Ainsi, l'apprentissage de la gestualité horlogère s'opère progressivement dans les dynamiques d'appropriation des outils et de manipulation des mouvements d'horlogerie auxquels les jeunes praticiens se trouvent confrontés. Les techniques corporelles se transmettent dans les usages que l'« agentivité » et les caractéristiques des objets manipulés impliquent. Le « geste juste », la technique adéquate se présentent rarement comme des modèles déjà constitués qu'il s'agirait de refaire, ils se déduisent du rapport que les apprentis instituent avec les objets qui balisent leur champ d'action et organisent leur intervention sur les « mouvements». C'est également dans les objets que s'actualisent les normes techniques qui façonnent l'activité, c'est à partir d'eux que la qualité du travail se déchiffre, se lit et se mesure ultérieurement, que le 
geste ou la technique d'approche se corrigent, parfois dans une analyse proposée par l'enseignant qui les décompose en plus petites séquences à des fins didactiques. En outre, si la gestualité technique se transmet, à l'école d'horlogerie, d'une volée d'apprentis à l'autre, c'est essentiellement parce qu'un matériel identique leur est fourni et qu'ils sont formés sur les mêmes objets, avec les mêmes outils. En circulant de la sorte, ces derniers favorisent la recréation d'usages similaires et l'apprentissage de références gestuelles qui doivent conduire à répondre aux mêmes objectifs techniques. Ces ensembles de gestes constituent alors un « répertoire » Geslin et Salembier, 2002 : 253) d'identification dont le partage graduel favorise l'émergence de sujets horlogers et figure les revendications d'appartenance commune à une même profession. Par ailleurs, de nombreux récits d'horlogers témoignent de l'importance de ces apprentissages par l'objet. À diverses reprises, certains praticiens m'ont conté leurs années de formation et leurs premières expériences professionnelles où ils se sont «faits la main » en soulignant l'importance de tous ces travaux de « rhabillage », de réparation de montres, voire de « restauration de pièces anciennes » dans l'acquisition de leur expérience technique. Dans ces contextes-ci, le métier s'apprend non directement d'un maître ou de l'imitation d'un tiers mais dans une sorte de «face-à-face » avec l'objet qui a bien souvent lieu en dehors du temps de travail et permet de rencontrer le plus grand nombre de pièces possibles, cristallisant ainsi différents cas de figures à identifier, diagnostiquer et « solutionner».

\section{Les jeux de voilement ou l'explicitation mise à mal}

La dernière partie de cet article sera consacrée à la caractérisation de certaines modalités de transmission du « sens mécanique » dans les écoles d'horlogerie. Cela me permettra de remettre en question la distinction anthropologique entre les formes scolaires et les formes initiatiques de transmission en montrant que l'injonction de dévoilement des manières de faire qui est souvent associée au paradigme pédagogique n'est pas nécessairement toujours mise en œuvre dans le cadre des écoles d'horlogerie. Parallèlement à cela, il s'agira de critiquer succinctement le caractère restrictif de la Convention qui fait de l'explicitation et l'objectivation formelle des savoir-faire une condition de leur transmission et de leur sauvegarde.

Dans une communication, l'anthropologue Daniel Fabre (2008) évoque la distinction anthropologique entre deux institutions de transmission des connaissances, à savoir l'école et l'initiation, qu'il distingue en relevant 
qu'elles fondent la diffusion des savoirs qu'elles produisent sur des impératifs différents : respectivement, la transparence et le voilement. Comme il le dit, « l'affirmation de l'institution de la forme scolaire dans la modernité occidentale s'est accompagnée d'un travail philosophique d'explicitation [je souligne] des opérations par lesquelles le sujet de raison se montre capable d'acquérir des connaissances en se constituant lui-même : c'est la pédagogie, [c'] est la science de la médiation » (Fabre, 2008:3). De manière contrastive, l'initiation est une forme de transmission qui joue sur les registres du secret et du masquage qui sont autant d' ' acte[s] dramatisé[s] [où l']on ne transmet pas [directement] un savoir dans la transmission mais [où l']on stabilise un statut et en même temps [l']on marque une césure temporaire [entre deux statuts, entre deux étapes]» (8). L'initiation se construit ainsi en imposant des mises à l'épreuve qui sont autant de rituels d'accès à ce qui est voilé (discours, savoirs, techniques). "Le paradoxe de l'initiation, qui est cognitivement presque impensable dans un monde de l'explication pédagogique, est que pour progresser, il faut régresser, pour avancer il faut redevenir un enfant [...] à qui on fait faire des actes qui n'ont aucune finalité [...] ». En ce sens c'est pour Fabre une « anti-pédagogie radicale» (8). Lors de mes premiers temps d'enquête dans le champ horloger de l'Arc jurassien, il me sembla que j'assistais à une actualisation effective de ce partage. Alors que les prises de contact avec le monde scolaire de la formation horlogère avaient été faciles et cordiales, j'avais évoqué avec plusieurs acteurs de l'« industrie » mon désir d'étudier les classes d'horlogerie des diverses écoles techniques afin d'y décrire la manière dont se transmettait le métier. L'un de mes interlocuteurs, « expert » dans une association de promotion de la " culture horlogère », me reprit un jour en disant : "à l'école, vous ne trouverez pas la transmission du savoir-faire horloger! ». Témoignant d'un élitisme assumé, il me signifiait que les lieux de formation « de base » que constituaient les écoles ne pouvaient être considérés comme dépositaires de l'« excellence » horlogère. Au monde de la «formation » répondait celui de la «profession » et seuls les « ateliers de fabrication » des montres constituaient de légitimes espaces de constitution et de transmission du savoir-faire. Mon interlocuteur s'empressa toutefois de me mettre en garde en relevant que « l'aura » de tels ateliers était voilée et qu'ils ne me seraient sans doute pas accessibles en vertu du « secret professionnel » et d'un principe «d'exclusivité ». S'est ainsi profilée la matrice discursive - qui devait, par la suite, se redéployer à de nombreuses reprises - de la « vraie » transmission horlogère : se déroulant dans l'espace confidentiel de l'atelier où les montres sont faites, ce type de transmission concernait un savoir d'initiés, authentique parce que « complet» et «complexe ». Ces 
considérations faisaient par ailleurs écho à des échanges que j'avais alors menés avec différent-e-s praticien-ne-s qui m'assuraient que, dans le milieu horloger, il est établi qu' " en sortant de l'école, un horloger ne sait pas travailler » et qu' « il lui faut dix années d'expérience, au bas mot, pour se déclarer vraiment horloger! » De tels discours sur la transmission de la «vraie » connaissance liaient ainsi inextricablement l'atelier de production, le monde professionnel, le secret et le savoir-faire, marquant ainsi ce dernier d'un sceau d'inaccessibilité, pour l'ethnologue que j'étais, du moins. Mon ethnographie de l'apprentissage horloger au sein des écoles professionnelles m'a néanmoins conduit à constater que ce partage entre formation/ profession calqué sur la dichotomie pédagogie/initiation ne résistait pas à l'examen critique pour différentes raisons. Tout d'abord, depuis plus d'une dizaine d'années, la formation horlogère peut être effectuée en mode dual. Les apprentis suivant les cours de théorie à l'école et les cours de pratique en entreprise, cela implique un important effort de coordination entre les entreprises et les écoles pour que le contenu des enseignements pratiques soient rigoureusement les mêmes, de part et d'autre. Les entreprises ont dès lors la responsabilité de former les apprentis en respectant un certain nombre de critères relatifs au règlement d'apprentissage de manière à les préparer adéquatement aux examens finaux qui se déroulent à l'école. Pour ce faire, les entreprises chargent généralement un de leurs horlogers de devenir « maître d'apprentissage » et par ce biais, élaborent tout un matériel didactique de description de leurs produits et d'explicitation des techniques présidant à leur fabrication. Il ne s'agit donc pas dans un univers où les secrets et les « couvertures sur la main » règnent en maîtres absolus. En deuxième lieu, j'ai observé que les pratiques de rétention d'informations, de mise sous silence de «trucs » faisaient intégralement partie des pédagogies de transmission du métier implémentées par les enseignants dans le cadre scolaire. Ainsi, dans ce qui suit, je désire montrer que l'institution scolaire chargée de l'apprentissage horloger en Suisse concentre une forme hybride de transmission qui ne correspond ni au paradigme pédagogique, ni au paradigme initiatique. J'en veux pour preuve différents exemples de didactique horlogère à l'œuvre dans les classes que j'ai eu l'occasion de suivre et d'observer. Les enseignants avec qui j'ai travaillé distinguaient ainsi trois « modèles » de transmission de la technique horlogère qu'ils mettaient tour à tour en œuvre en fonction des objectifs du module dispensé et du temps qu'ils avaient à disposition. En premier lieu, il y a le modèle de la « démonstration », de la « décomposition du geste » où l'enseignant inaugure chaque exercice en donnant un « exemple » à la classe d'apprentis. Il leur «montre » ainsi ce qui est « attendu » d'eux en « décomposant » 
toutes les étapes qui composent les différentes opérations à réaliser et en distinguant les différentes séquences de gestes et d'attentions qu'elles requièrent. Il caractérise explicitement les problèmes, rend la classe attentive à la localisation et à la teneur des difficultés qu'elle sera amenée à rencontrer, par exemple, en démontant ou en remontant tel ou tel mouvement d'horlogerie qui a ses particularités propres. Bien que, dans ce modèle-ci, les enseignants ne soient pas réticents à redire les informations, à remontrer la procédure des opérations, cette didactique-là ne donne pas souvent lieu à des formes de répétition. À ce titre, un enseignant me glissait : « les élèves qui sont ici, ils ne sont pas là pour rien. Pas besoin de répéter deux fois les choses, je le ferais si besoin était, mais ils fonctionnent énormément par mimétisme... tu fais les choses une fois devant eux, ils le voient et après ça, généralement ils ont compris, ils ne reviennent pas demander. Ils captent vite et sont capables de trouver une manière de refaire ! » Il est intéressant de constater que sans qu'il s'agisse d'une norme explicite devant inciter les apprentis à être immédiatement attentifs pour ne pas avoir besoin de revenir demander de l'aide, ceux-ci sont toutefois valorisés pour la rapidité de leur assimilation et leur aptitude à l'autonomie. En second lieu, se trouve une déclinaison particulière de ce type de transmission par « monstration » mais cette fois-ci, elle n'est pas mise en œuvre à titre d'exemple « pour toute la classe » et n'est accompagnée d'aucune explicitation verbale. Ce qui est montré à l'élève ne fait l'objet d'aucune thématisation : l'apprentissage demeure indéniablement tacite et enjoint à la « débrouille ». Enfin, un autre cas d'étude de la transmission horlogère au sein de l'institution scolaire semble ici intéressant en ce qu'il conteste, sinon remet en cause l'acception selon laquelle le modèle pédagogique organise la formation en école à partir d'un impératif de transparence. L'apprentissage du métier n'est pas uniquement axé sur la « démonstration » des gestes ou des techniques adéquates, à répéter par imitation. Ces formes de mimétisme présentent, aux dires de certains enseignants, d'évidentes limites car elles ne conduisent pas toujours les apprentis à développer leur «sens mécanique » qui comme nous l'avons vu, suppose l'incorporation d'un important principe d'autonomie. Aussi, la transparence pédagogique présumée se défait. Au modèle de la «monstration » se substitue celui de l'incitation à la « démerde » et à l'apprentissage de l'« observation ». L'injonction de dévoilement des manières de faire auquel on lie la pédagogie et à partir de laquelle l'apprentissage dans le cadre scolaire est souvent pensé s'obscurcit. Certains enseignants horlogers admettent alors que pour mieux transmettre, il faut parfois préalablement voiler l'accès à certains trucs en gardant le silence, 
en ne disant rien, en ne donnant aucunes informations aux élèves. L'apprentissage du métier est ainsi, en partie, conditionné par un autre type de didactique scolaire qui conduit à la mise en œuvre de jeux de voilement des solutions, du savoir horloger et de la gestualité dans laquelle il s'incarne. Les apprentis doivent ainsi explorer l'accès au « comment faire ? " par d'autres voies. Il ne s'agit pas, à proprement parler, de secret, ou plutôt par secret, il ne faut pas entendre ici l'empêchement total d'accès à une information mais un masquage provisoire de cet accès. Ces pratiques de voilement ne le restreignent jamais définitivement mais le diffèrent dans le temps pour «inciter les apprentis à l'observation ». De cette façon, s'établit l'instruction relative aux techniques du regard et à l'apprentissage $\mathrm{du}$ « savoir voir ». À travers ce silence calculé, se profile une volonté d'agir sur les actions des élèves en les contraignant à acquérir une capacité à trouver et résoudre un large spectre de problèmes sans avoir recours à « l'aide du prof' ». Ils doivent apprendre à constituer leurs ressources «par euxmêmes », à partir de formalisations de procédures existantes ou non, et à mettre en place leur propre méthodologie de travail en consignant les «problèmes rencontrés » dans un « journal de bord ». Dans ce modèle-ci, la communication à l'atelier n'est pas rompue, l'économie de la parole s'organise seulement selon un autre canevas. L'« envie de transmettre » n'est pas davantage exclue de ce processus de voilement, ce dernier en est, au contraire, une des modalités. Il a pour fonction d'optimiser l'apprentissage technique et d'inscrire les apprentis dans un régime de valeurs qu'ils doivent faire leurs. Ce qui est ainsi transmis se condense dans une forme de contrainte à la « démerde », autrement dit à l'incorporation d'un principe d'autonomie dans le travail. À cet égard, l'apprentissage est parfois vécu, tant du côté des maîtres que des apprentis, comme un combat et une mise à l'épreuve. Cela rejoint certaines observations que j'ai pu réaliser chez un maître-horloger indépendant qui recevait une fois par semaine à son atelier un jeune apprenti de l'école d'horlogerie et disait à son horloger alors chargé d'encadrer le nouveau venu : « non, non, ne lui en dis pas trop, ne lui mâche pas le travail! » Ces temps d' " anti-pédagogie » pédagogique créent les cadres pour que se déploie une imprégnation au métier qui demande toutefois du temps et supporte mal les phases de « coup de bourre », pourtant nombreuses tout au long de l'année scolaire, où il faut « gagner du temps » et où les apprentis doivent alors « avancer ». L'institution scolaire liée à la formation professionnelle et le modèle pédagogique de transmission qu'elle incarnerait prétendument n'est, en définitive, pas un lieu de transparence par excellence. Elle demeure traversée, de part en part, par des logiques de voilement, des stratégies de rétention, de masquage des informations ou de 
dissimulation des solutions. Ces pratiques ont cependant une durée limitée et l'intensité avec laquelle elles sont maintenues dépend du temps que les enseignants ont à disposition pour explorer un module. Elles sont des mises à l'épreuve qui constituent autant de moyens de transmettre le métier et de faire des apprentis horlogers, des professionnels. Ces considérations rejoignent, par ailleurs, la conclusion à laquelle parvient Fabre qui ne s'en tient pas, à la fin de sa communication, à cette stricte dualité entre pédagogie et initiation, et montre la porosité de ce partage. Il relève ainsi que « les institutions qui pensent tenir les moyens de la transparence de l'éducation, de la reproduction de la société, laissent place dans le secret, la marge, à des pratiques qui relèvent très explicitement de la logique initiatique, s'inspirant même parfois des mondes où l'initiation est centrale pour la production des individus pleinement socialisés »(Fabre, $2008: 9$ ).

Ces différents exemples tendent à prouver que les formes de transmission et d'apprentissage horlogers ne font pas systématiquement l'objet d'explicitations, même s'ils sont conscients et réfléchis. Ceci remet fortement en question la pertinence de la Convention qui, tout en relevant que la transmission est l'étape préalable de tout processus de sauvegarde, n'en considère pas moins que ce qui se transmet est toujours explicité, ou du moins explicitable. Dans un certain nombre de cas toutefois, la transmission des compétences horlogères et des valeurs qui les façonnent se réalise justement dans des formes de voilement. La Convention, qui ne repère que les savoir-faire identifiés et revendiqués comme tels, semble ainsi bien incapable de la prendre en charge. Cette transmission tient en effet parfois son efficacité de la mise sous silence provisoire de certains principes, astuces ou techniques qui ont trait au métier. Considérant que l'apprentissage scolaire des habiletés horlogères s'opère également dans ces demi-revers de la visibilité, il apparaît que la formalisation qu'implique sa qualification patrimoniale se « cogne ", en définitive, à tout ce qui, dans la circulation du «sens mécanique », n’est pas révélé.

\section{Conclusion. La transmission du " sens mécanique " horloger au risque de sa mise en patrimoine}

Au vu de ce qui précède, la Convention ne peut pas constituer un moyen de transmission du métier d'horloger, ne serait-ce que parce que le programme de sauvegarde et de lutte contre les menaces de perte qui pèsent sur les pratiques sociales qu'elle implique se résume à un processus d'identification et d'inventorisation de celles-ci sous formes de fiches et de listes. Une activité technique n'est, au demeurant, pas réductible à la 
somme des connaissances et des composantes matérielles qu'elle mobilise, fussent-elles identifiables, ce qui est loin d'être toujours le cas. De plus, faire l'inventaire des «ingrédients » qu'une telle pratique requiert ne permet pas de reconstituer rétroactivement les processuss cognitifs qui la sous-tendent. Les dimensions inexorablement incorporées et distribuées de la cognition horlogère demeurent difficilement inventoriables pour quels que projets de captation patrimoniale que ce soient. Les dynamiques de mise en œuvre et de transmission du « sens mécanique » horloger sont, en effet, des processus complexes, diffus, en partie informels (et difficilement formalisables) qui ne résistent pas à la traduction simplificatrice et réductrice qu'une telle patrimonialisation opère inévitablement. La captation du « sens mécanique » et son objectivation en PCI à des fins de sauvegarde auraient assurément pour effet paradoxal de ne pas satisfaire les conditions propices à sa transmission. Bien que le paradigme de $\mathrm{PCI}$ réserve une importante place à cette notion, il semble cependant constituer un cadre artificiel pour la prise en charge des pratiques de savoir en horlogerie. La Convention peut être un indéniable instrument de promotion de l'industrie horlogère suisse dans le monde, elle peut mettre en valeur ses produits, ses acteurs et ses territoires, susciter des projets d'exposition, inciter à la relève, témoigner de l'importance de cette activité pour l'économie helvétique, mais elle ne peut avoir, en définitive, la prétention de constituer un opérateur de transmission de la technique horlogère. 


\section{Références}

Adell, Nicolas, 2010, « Argumentaire scientifique » préparatoire au colloque international Transmettre : quel(s) patrimoine(s) ? Autour du Patrimoine Culturel Immatériel, Toulouse, Université de Toulouse II - Le Mirail, 16-18 juin 2010.

Chevallier, Denis et Chiva, Isac, 1991, «L'introuvable objet de la transmission ». Dans Denis Chevallier (dir.), Savoir faire et pouvoir transmettre: transmission et apprentissage des savoir-faire et des techniques : 1-11. Paris, Editions de la Maison des Sciences de l'Homme.

Cornu, Roger, 1991, «Voir et savoir ». Dans Denis Chevallier (dir.), Savoir faire et pouvoir transmettre: transmission et apprentissage des savoir-faire et des techniques : 83-100. Paris, Editions de la Maison des Sciences de l'Homme.

Downey, Greg, 2007, « Seeing without Knowing, Learning with the Eyes: Visuomotor 'Knowing' and the Plasticity of Perception ». Dans Mark Harris (dir.), Ways of Knowing: New Approaches in the Anthropology of Knowledge and Learning : 222-241. New York et Oxford, Berghahn Books.

Fabre, Daniel, 2007, «Genèses de l'anthropologie : le paradigme des derniers ». Cours donné dans le cadre du Séminaire du LAHIC : L'institution de la culture, 2007-2010. Paris, LAHIC.

Fabre, Daniel, 2008, « À propos de la forme scolaire de transmission ». Contribution présentée le 24 janvier 2008, dans le cadre du Séminaire du LAHIC (Laboratoire d'anthropologie et d'histoire de l'institution de la culture) : L'institution de la culture, 2007-2008. Paris, LAHIC.

Ingold, Tim, 2000, The Perception of the Environment. Essays in Livehood, Dwelling and Skill. London \& New York, Routledge.

Geslin, Philippe et Salembier Pascal, 2002, «La fleur, l'épice : usage social du corps et communauté de pratique dans la relance de produits "authentiques" ». In Blandine Bril et Valentine Roux (dir.), Le geste technique: réflexions méthodologiques et anthropologiques : 253-269. Ramonville Saint-Agne, Erès.

Gonseth Marc-Olivier et al. (dir.), 2011. Bruits : échos du patrimoine culturel immatériel. Neuchâtel : Musée d'ethnographie de Neuchâtel (MEN).

Grasseni, Cristina, 2004, «Skilled vision. An apprenticeship in breeding aesthetics », Social Anthropology 12(1) : 41-55.

Hafstein, Valdimar Tr., 2009, «Intangible Heritage as a List: From Masterpieces to Representation ». Dans Laurajane Smith et Natsuko Akagawa (dir.), Intangible Heritage : 93-111. London and New York: Routledge. 
Lave, Jean, 2011, Apprenticeship in critical ethnographic practice. Chicago, University of Chicago Press.

Leroi-Gourhan, André, 1981, Le geste et la parole 1 : Technique et langage. Paris, Albin Michel.

Marti, Laurence, 2004, Participation et non-participation à la formation continue professionnelle : l'exemple des horlogers et horlogères ainsi que des cuisiniers et cuisinières. Bern, Direction du programme PNR 43.

Munz, Hervé, 2011, «L'artisanat traditionnel dans l'horlogerie suisse: une catégorie introuvable? », Folklore suisse, Bulletin de la Société suisse des traditions populaires, 101(1) : 8-13.

Munz, Hervé et Geslin Philippe, 2010, « Le patrimoine culturel immatériel à l'épreuve des savoir-faire horlogers de l'Arc Jurassien », Museums 5 : 26-29.

Pelegrin Jacques, 1991, «Les savoir-faire : une très longue histoire », Terrain $16: 106-113$.

UNESCO, 2003, Texte de la Convention pour la sauvegarde du patrimoine culturel immatériel, $32^{\mathrm{e}}$ session de la Conférence générale de l'UNESCO, 29 septembre-17 octobre 2003. Paris : UNESCO. 